\title{
Polarimetric studies of comet Levy 1990 XX
}

\author{
H. S. Das ${ }^{1}$ and A. K. Sen ${ }^{2, \star}$ \\ 1 Department of Physics, Karimganj College, Karimganj 788710, Assam, India \\ e-mail: hs_das@rediffmail.com \\ 2 Department of Physics, Assam University, Silchar 788011, Assam, India \\ e-mail: asokesen@sancharnet.in \\ Received 23 April 2005 / Accepted 11 August 2006
}

\section{ABSTRACT}

\begin{abstract}
The spherical and non-spherical dust-grain characteristics of comet Levy $1990 \mathrm{XX}$ are studied in this paper. Using T-matrix theory, a good theoretical fit to the polarisation data observed by Chernova and co-workers for comet Levy $1990 \mathrm{XX}$ is reported in the present work. The calculated degree of linear polarisation $(p)$ as a function of observed scattering angle $(\theta)$ produced by prolate spheroidal crystalline olivine particles with effective radius $\left(r_{\text {eff }}=0.218 \mu \mathrm{m}\right)$, effective variance $\left(v_{\text {eff }}=0.0036\right)$, and aspect ratio $(E=0.486)$ are compatible with the comet's observed polarisation at $\lambda=0.485 \mu \mathrm{m}$. After comparing the above result with the Mie theory result ( $r_{\mathrm{eff}}=0.115 \mu \mathrm{m}, v_{\mathrm{eff}}=0.0465$ ), it is found that prolate grains give the better fit to the observed polarisation data. Also, the negative polarisation behaviour of comet Levy $1990 \mathrm{XX}$ is discussed.
\end{abstract}

Key words. comets: general - dust, extinction - scattering - polarization

\section{Introduction}

Polarimetry in the continuum is a good technique for studying the nature of cometary dust grains. Many authors (Bastien et al. 1986; Kikuchi et al. 1987, 1989; Lamy et al. 1987; Le Borgne et al. 1987; Mukai et al. 1987; Sen et al. 1991a,b; Das et al. 2004 , etc.) have studied the linear and circular polarisation measurements of several comets. Since the last apparition of comet Halley, observers have been using a set of filters (centred at $\lambda=0.365 \mu \mathrm{m}, 0.485 \mu \mathrm{m}$, and $0.684 \mu \mathrm{m}$ ) known as IHW (International Halley Watch) filters to avoid contamination by line emission.

The space missions to comet Halley made useful in situ measurements and have given important information about the grainmass distribution (Mazets et al. 1987). Lamy et al. (1987) derived the grain-size distribution for Halley by comparing the data from space crafts Vega I, Vega II, and Giotto and the same grainsize distribution has been used by Das et al. (2004) to explain the polarimetric behaviour of comet Halley and other comets.

Infrared emission from comets also gives valuable information about the composition of cometary grains and their size (Gehrz \& Ney 1992; Hanner et al. 1994). It has been found that comet Levy 1990 XX displays a strong silicate feature with a distinct peak at $11.25 \mu \mathrm{m}$, attributed to crystalline olivine grains (Lynch et al. 1992).

Das et al. (2004) discussed the grain aging of comets by solar radiation for four non-periodic comets (Hyakutake, Austin, Bradfield, Levy 1990 XX). The authors found an empirical relation between the relative abundance of coarser grains' index $(g)$ and perihelion distance $(q)$ of the form $g=-2.5 q^{2 / 3}$ and commented that comets whose grains are processed more by the solar radiation do contain a relatively smaller number of finer grains. From the work of Das et al. (2004), it was found that the grains of comet Levy $1990 \mathrm{XX}$ are much smaller, as compared to the grains of Hyakutake, Austin, Bradfield, Hale-Bopp, and Halley.

^ also Associate, Inter University Centre for Astronomy and Astrophysics (IUCAA), Ganeshkhinde, Pune 411007, India.
It is now generally accepted that, cometary grains are not spherical and may be irregular or spheroidal in shape. The spherical grain characteristics of comets can be studied using the Mie scattering theory. One can find polarisation values for light scattered by compact spheres using this theory and can match the result with observed polarisation data (Sen et al. 1991a,b; Das et al. 2004). But cometary particles are "fluffy aggregates" or are porous, with irregular shapes (Greenberg \& Hage 1990). The measuring circular polarisation of comet Hale-Bopp (Rosenbush et al. 1997) also reveals that cometary dust grains must be composed of non-spherical particles. Xing \& Hanner (1997) carried out calculations with porous grains of different shapes and sizes with discrete dipole approximation (DDA) method. In order to study the irregular grain properties of comets, T-matrix theory (Mishchenko 1991, 1998) has been used by many investigators (Kolokolova et al. 1997; Kerola \& Larson 2001). Using T-matrix theory, Kerola \& Larson (2001) calculated polarisation for non-spherical particles and compared the results with the polarimetric measurements of comet Hale-Bopp. They found that the prolate grains can explain the observed polarisation in a more satisfactory manner compared to the other shapes.

In this paper, the irregular grain properties of comet Levy 1990 XX have been studied using Mishchenko's (1991, 1998) T-matrix code. The results obtained from the T-matrix theory are compared with Mie theory results. The negative polarisation behaviour of comet Levy $1990 \mathrm{XX}$ is also discussed.

\section{Grain characteristics of comet Levy 1990 XX}

The observed linear polarisation of comets is generally a function of $(i)$ the wavelength of incident light $(\lambda)$, (ii) the scattering angle, $\theta\left(=180^{\circ}\right.$ - Phase angle), (iii) the geometrical shape and size of the particle, (iv) the composition of dust particles in terms of the complex values of the refractive index, $m(=n-i k)$. The shape and size of a spheroid can be specified by the axial ratio, $E(=a / b)$, and the equal- surface-area-sphere radius, $r_{\mathrm{s}}$ (or the equal-volume-sphere radius, $r_{v}$ ). The axial ratio $E>1$ for oblate spheroids, $E<1$ for prolate spheroids and $E=1$ 
for spheres. The polarimetric data of comet Levy 1990 XX was taken from Chernova et al. (1993). Since the polarimetric data is only available at $\lambda=0.485 \mu \mathrm{m}$, our analysis is restricted to that wavelength. Using the T-matrix theory, Kerola \& Larson (2001) calculated polarisation for irregular particles and compared the results with the observed polarimetric data of comet Hale-Bopp. They achieved reasonably good agreement with one set of spherical volume element values of effective radius $\left(r_{\mathrm{eff}}\right)$, effective variance $\left(v_{\mathrm{eff}}\right)$, and $E(0.216 \mu \mathrm{m}, 0.0105$ and 0.415 respectively $)$ for prolate spheroids at $\lambda=0.485 \mu \mathrm{m}$ and $\lambda=0.684 \mu \mathrm{m}$. Since olivine grains have been detected in comet Levy 1990 XX (Lynch et al. 1992), one can assume that the index of refraction is given by that of olivine $(1.63,0.00003)$.

After this analysis of the polarimetric data for comet Levy 1990XX using both the Mie and T-matrix theories, we compare the results with the two theories below.

\subsection{The Mie theory}

The Mie theory provides an analytic solution to the scattering problem for spheres and correctly describes the interaction of light for homogeneous spherical particles of any size in general. Several investigators (Mukai et al. 1987; Sen et al. 1991a,b; Chernova et al. 1994; Joshi et al. 1997; Kiselev \& Velichko 1998) have studied different comets and tried to analyse the dust grain behaviour of comets using the Mie theory. The power law size distribution has been used for the analysis of polarimetric data, where the minimum and maximum particle radii are automatically fixed for each and every run merely by specifying the particle's effective radius $\left(r_{\text {eff }}\right)$ and effective variance $\left(v_{\text {eff }}\right)$. The best-fit polarisation values obtained from the present work at $\lambda=0.485 \mu \mathrm{m}$ are $r_{\mathrm{eff}}=0.115 \mu \mathrm{m}, v_{\mathrm{eff}}=0.0465$, and the $\chi^{2}$-value for this analysis is found to be 41.6. Also in the present study, several other combinations involving smaller sizes $\left(0.10 \mu \mathrm{m} \leq r_{\mathrm{eff}} \leq 0.12 \mu \mathrm{m}\right)$ and a range of variances were tried. But, none of these combinations allowed an overall fitting to the observed data of comet Levy 1990XX. However, it can be seen from Fig. 1 that the calculated polarisation curve ( $r_{\text {eff }}=0.115 \mu \mathrm{m}, v_{\text {eff }}=0.0465$ ) shows a poor fit to the observed data, so it can be inferred that the dust grains in comet Levy's coma are not perfectly spherical.

\subsection{The T-matrix theory}

The T-matrix theory is a powerful technique for studying the non-spherical grain properties in comets. In this paper, calculations were carried out for randomly oriented spheroids using Mishchenko's (1998) single-scattering T-matrix code, which is available at http://www.giss.nasa.gov/ crmim. The best way to execute the T-matrix program is to use the power-law size distribution. The main feature of the T-matrix approach is that it reduces exactly to the Mie theory when the particle is a homogeneous or layered sphere composed of isotropic materials.

Kerola \& Larson (2001) studied comet Hale-Bopp using the T-matrix theory and found best-fit parameters at $\lambda=0.485 \mu \mathrm{m}$ and $\lambda=0.684 \mu \mathrm{m}$ using prolate spheroids $\left(r_{\mathrm{eff}}=0.216 \mu \mathrm{m}\right.$, $\left.v_{\text {eff }}=0.0105, E=0.415\right)$. The result for oblate spheroids is also reported by them, but the result agrees well in the red wavelength but not simultaneously in blue.

The best-fit polarisation values obtained from the present work at $\lambda=0.485 \mu \mathrm{m}$ are $r_{\text {eff }}=0.218 \mu \mathrm{m}, v_{\text {eff }}=0.0036$, and $E=0.486$. Using these parameters, a theoretical polarisation curve was generated using the T-matrix code and is plotted in Fig. 1. The $\chi^{2}$-value for this analysis is 5.22. Thus, it can be seen

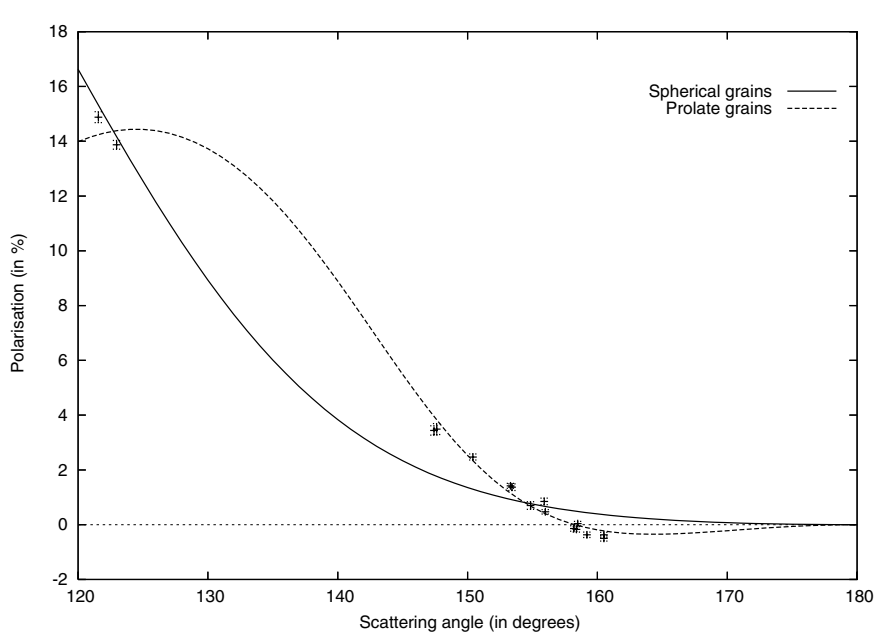

Fig. 1. Comparison of the results from the Mie and T-matrix theories. The solid line and the dotted line represent the best-fit polarisation values for spherical grains and prolate grains obtained from the Mie and the T-matrix theories, respectively. The Mie theory and T-matrix theory fits use different particle shapes and size ranges as explained in the text.

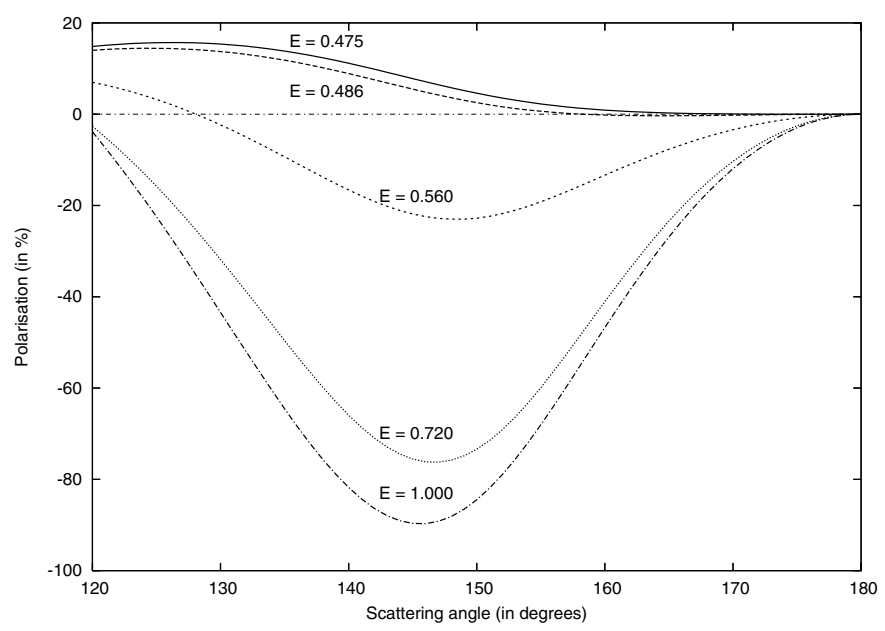

Fig. 2. Comparisons of T-matrix calculations using prolate spheroid grains having effective radius, $r_{\mathrm{eff}}=0.218 \mu \mathrm{m}$ and effective variance, $v_{\text {eff }}=0.0036$ for different values of $E(=0.475,0.486,0.560,0.720)$ of the particle. A curve using the same size distribution for Mie particles (i.e., $E=1.000$ ) is also shown.

that the polarisation curve for prolate grains agrees well with the observed data. But, no such good fit has been found for oblate grains.

Figure 2 shows the polarisation curves at $\lambda=0.485 \mu \mathrm{m}$ using prolate spheroid grains having $r_{\text {eff }}=0.218 \mu \mathrm{m}$ and $v_{\text {eff }}=0.0036$ for different values of $E$ for the particle. It can be seen that the curves change for an assortment of $E$ values for prolate spheroids. A curve using the same size distribution for Mie particles (i.e., $E=1.000$ ) is also shown there.

At this stage, one may note that the fit with the T-matrix theory is for a very specific prolate shape $(E=0.486)$ for all the grains and that their size is very closely centred on $r_{\mathrm{eff}}=0.218 \mu \mathrm{m}$. In reality, the size distribution measured by spacecrafts for comet Halley (Mazets et al. 1987) was found to be much broader than the size range obtained from the present fit. However, the fit obtained is good, and in that sense, it is possible to reproduce the negative polarisation branch of comets with prolate grains having these very tight constraints. 
It can be seen from Fig. 2 that there is negative polarisation at the level of about $-90 \%$ produced by spherical particles $(E=1)$ at a scattering angle of about $145^{\circ}$ having the size distribution parameter: $r_{\text {eff }}=0.218 \mu \mathrm{m}$ and $v_{\text {eff }}=0.0036$. This result is a little unusual, since the Mie theory produces positive polarisation with a peak around 90 to 120 degrees in phase angles (or 90 to 60 degrees in scattering angles) and a negative polarisation branch above about 160 degrees for the scattering angle.

Actually, the discrepancy in Fig. 2 (for $E=1$ ) arises due to the use of a different size distribution parameter. Section 2.1 already discusses that by using the Mie theory the best-fit parameter for spherical grains can only be achieved at $r_{\mathrm{eff}}=0.115 \mu \mathrm{m}$ and $v_{\text {eff }}=0.0465$. Other than this combination of parameters, the Mie theory does not give any satisfactory result, so for this particular size distribution parameter $\left(r_{\mathrm{eff}}=0.218 \mu \mathrm{m}\right.$, $v_{\text {eff }}=0.0036$ ), prolate grains show a more satisfactory result. Thus it is clear from Fig. 2 that, when the value of $E$ decreases, the polarisation curve shifts towards the observed data points and finally shows the best-fit at $E=0.486$.

The simulation to reproduce the observed polarisation of comet Hale-Bopp as done by Kerola \& Larson (2001) produced a value of $-85 \%$ negative polarisation (never observed in reality) by spherical particles at a scattering angle of about $125^{\circ}$ for $r_{\text {eff }}=0.216 \mu \mathrm{m}$ and $v_{\text {eff }}=0.0105$. However, the best-fit was found only at $E=0.415$.

Greenberg \& Li (1996) studied interstellar dust polarisation and found that prolate grains can give more satisfactory results as compared to other shapes. Actually, prolate spheroids are a natural result of the process of clumping in the proto-solar nebula (Kerola \& Larson 2001). Thus it has been seen that prolate grains can give more satisfactory results for comet Hale-Bopp and also for comet Levy 1990 XX.

\section{Discussions}

The T-matrix theory is a powerful tool for studying the polarimetric behaviour of comets with non-spherical grains, which means that the Mie theory will give less accurate results than the T-matrix theory if grains are not spherical. Also, the $\chi^{2}$-value emerging from the Mie theory is 41.6 and the T-matrix theory is 5.22. It is thus clear that T-matrix calculation gives a better fit to the observed data. In Fig. 1, the expected polarisation curve is plotted on the observed data points for both spherical grains (based on the Mie theory) and prolate grains (based on the $\mathrm{T}$-matrix theory). Thus one can see that prolate grains can give more satisfactory results for comet Levy 1990 XX. Cometary grains can also, have other shapes but a simple model is considered in the present work.

The negative polarisation behaviour of a comet is very interesting. Many comets show negative polarisation beyond the $157^{\circ}$ scattering angle (Kikuchi et al. 1987; Chernova et al. 1993; Ganesh et al. 1998, etc.). Several investigators (Greenberg \& Hage 1990; Muinonen 1993) have discussed the cause of negative polarisation in comets. The mechanism of coherent back scattering proposed by Muinonen (1993) was used to explain the negative polarisation. The fluffy aggregate model originally proposed by Greenberg \& Hage (1990) and later adopted by Xing \& Hanner (1997) is also preferred for studying negative polarisation in comets. Many investigators (Mukai et al. 1987; Sen et al. 1991a,b; Joshi et al. 1997) generated the expected polarisation curve using the Mie theory that shows negative polarisation beyond $157^{\circ}$. It is to be noted that the results obtained from the T-matrix code could not reproduce the negative polarisation branch observed for comet Hale-Bopp, as seen in
Kerola \& Larson (2001), where the analysis was restricted to $\theta \leq 160^{\circ}$. They also concluded that a combination of viewing geometry effects and enhanced multiple scattering might provide a quantitative explanation of the negative polarisation beyond $160^{\circ}$. In the present work, it is also interesting to note that the Tmatrix theory can reproduce the negative polarisation observed in comet Levy 1990 XX, which Mie can't. With a very restrictive set of parameters $r_{\mathrm{eff}}=0.218 \mu \mathrm{m}, v_{\mathrm{eff}}=0.0036$, and $E=0.486$ at $\lambda=0.485 \mu \mathrm{m}$, one can generate a negative polarisation curve using T-matrix theory for $\theta \geq 157^{\circ}$. But it is also important to study the fluffy grains with irregular shapes and enhanced multiple scattering, which may explain the negative polarisation well in comets, but a systemic approach in this direction was beyond the scope of the present work.

\section{Conclusions}

1. The best-fit parameters discovered for the observed polarisation data of comet Levy, at $\lambda=0.485 \mu \mathrm{m}$ for prolate spheroids, are $r_{\mathrm{eff}}=0.218 \mu \mathrm{m}, v_{\mathrm{eff}}=0.0036$, and $E=0.486$.

2. The $\chi^{2}$-value for the T-matrix theory is 5.22, whereas the $\chi^{2}$ value is 41.6 for the Mie theory. Thus one can say that the prolate grains give a better fit to the observed polarisation data.

3. The expected negative polarisation values have been successfully generated for comet Levy 1990 XX using the T-matrix theory.

Acknowledgements. The author AKS thanks the Dept. of Space, Govt. of India, for financial assistance under the RESPOND research project to carry out this work. The authors are very thankful to the anonymous referee of this paper for valuable suggestions and comments, which greatly helped to improve the quality of this paper.

\section{References}

Bastien, P., Menard, F., \& Nadeau, R. 1986, MNRAS, 223, 827 Chernova, G. P., Kiselev, N. N., \& Jockers, K. 1993, Icarus, 103, 144 Das, H. S., Sen, A. K., \& Kaul, C. L. 2004, A\&A, 423, 373 Delsemme, A. H. 1987, in Symposium on diversity and similarity of comets, ed. E. J. Rolfe, \& B. Battrick, ESA, Garching, ESA SP -278, 19

Eaton, N., Scarrott, S. M., \& Gledhill, T. M. 1992, MNRAS, 258, 384 Ganesh, S., Joshi, U. C., Baliyani, K. S., \& Deshpande, M. R. 1998, A\&AS, 129, 489

Gehrz, R., \& Ney, E. 1992, Icarus, 100, 162

Greenberg, J. M., \& Hage, J. I. 1990, ApJ, 361, 260

Greenberg, J. M., \& Li, A. 1996, A\&A, 309, 258

Hanner, M. S., Lynch, D. K., \& Russell, R. W. 1994, ApJ, 425, 275

Joshi, U. C., Baliyan, K. S., Ganesh, S., et al. 1997, A\&A, 319, 694

Kerola, D. X., \& Larson, S. M. 2001, Icarus, 149, 351

Kikuchi, S., Mikami, Y., Mukai, T., Mukai, S., \& Hough, J. H. 1987, A\&A, 187, 689

Kikuchi, S., Mikami, Y., Mukai, T., \& Mukai, S. 1989, A\&A, 214, 386

Kiselev, N. N., \& Velichko, F. P. 1998, Icarus, 133, 286

Kolokolova, L., Jockers, K., \& Chernova, G. 1997, Icarus, 126, 351

Lamy, P. L., Grün, E., \& Perrin, J. M. 1987, A\&A, 187, 767

Le Borgne, J. F., Leroy, J. L., \& Arnaud, J. 1987, A\&A, 187, 526

Levasseur-Regourd, A. C., Hadamcik, E., \& Renard, J. B. 1996, A\&A, 313, 327

Lynch, D., Russel, R., Hackwell, J., Hanner, M., \& Hammel, H. 1992, Icarus, 100,197

Manset, N., \& Bastien, P. 2000, Icarus, 145, 203

Mazets, E. P., Sagdeev, R. Z., Aptekar, R. L., et al. 1987, A\&A, 187, 699

Mishchenko, M. I. 1991, J. Opt. Soc. Am. A, 8, 871

Mishchenko, M. I., \& Travis, L. D. 1998, J. Quant. Spect. Rad. Transf., 60, 309

Muinonen, K. 1993, in Asteroids, Meteors, Comets, IAU Symp., 160, 271

Mukai, T., Mukai, S., \& Kikuchi, S. 1987, A\&A, 187, 650

Rosenbush, V. K., Shakhovskoj, N. M., \& Rosenbush 1997, earth, Moon \& Planets, 78, 381

Sen, A. K., Deshpande, M. R., Joshi, U. C., Rao, N. K., \& Raveendran, A. V. 1991a, A\&A, 242, 496

Sen, A. K., Joshi, U. C., \& Deshpande, M. R. 1991b, MNRAS, 253, 738

Xing, Z., \& Hanner, M. S. 1997, A\&A, 324, 805 